\title{
La tasa acumulada de recién nacido vivo aumentaría a mayor número de intentos de fertilización in vitro
}

\author{
The cumulative rate of live birth would increases with the number of in vitro fertilization attempts
}

\section{Objetivos}

Determinar la tasa de recién nacido vivo (RNV) por ciclo iniciado de fertilización in vitro (FIV) y la tasa acumulada de RNV en subsiguientes ciclos.

\section{Diseño, lugar y pacientes}

Estudio de cohorte prospectivo, realizado en el Reino Unido, que incluyó a 156.947 mujeres que realizaron 257.398 ciclos de FIV, entre el año 2003 y 2010 y que fueron seguidas hasta el año 2012. Se definió ciclo de FIV a cada episodio de estimulación ovárica, con sus subsecuentes embriotransferencias, ya sea en ciclos frescos y congelados. La edad media de inicio del tratamiento fue de 35 años y la duración media de la infertilidad fue de cuatro años. Fueron excluídos tratamientos para criopreservación de ovocitos, donación de ovocitos o subrogación uterina.

\section{Evaluación de factores pronósticos}

Edad en mujeres que utilizaron ovocitos propios: < 40, 40 a 42 y $>42$ años. Fuente de ovocitos: propios vs donados. Tipo de tratamiento: ICSI (del inglés, inyección intracitoplasmática de espermatozoides), semen de donante.

\section{Medición de resultados principales}

Se determinó como resultado principal la tasa de RNV por ciclo
Smith AD, y col. JAMA 2015;314(24):2654-2662. de FIV y tasa acumulada de RNV en todos los ciclos realizados. La tasa acumulada fue calculada estimando diferentes escenarios posibles de continuidad del tratamiento, según como se asumía la tasa potencial de RNV en las mujeres que abandonaban el tratamiento prematuramente: a) Optima: asumió una tasa de RNV similar a aquellas mujeres que continuaban con los intentos; b) Conservadora: asumió una tasa de RNV igual a cero; c) Ajustada a pronóstico: asumió una tasa de RNV en un punto intermedio (más realista), con un valor igual a cero en pacientes catalogadas con mal pronóstico (escaso recupero de ovocitos y mayor edad al primer ciclo), o un mayor valor (similar al de las mujeres que continuaban con los intentos), en aquellas que abandonaban por otras causas (ej. dificultades económicas, estrés emocional, emigración). El análisis se realizó por intención de tratar.

\section{Resultados Principales}

Los resultados principales se resumen en la tabla 1. En mujeres que usaron ovocitos propios las tasas de RNV por edad, en el primer y sexto ciclo, fueron: < 40 años, $32,3 \%$ y $68,4 \%$; entre 40 a 42 años, $12,3 \%$ y $31,5 \%$; y $>42$ años, todas las tasas fueron $<4 \%$. No hubo diferencias por edad en pacientes que recibieron ovocitos donados. Las tasas de RNV fueron menores en pacientes con factor masculino sin tratamiento, sin embargo con ICSI y semen de donante desaparecieron estas diferencias.

Tabla 1. Tasa de RNV por ciclo iniciado y tasa acumulada de RNV en ciclos repetidos

\begin{tabular}{|c|c|c|c|c|c|}
\hline \multirow[b]{2}{*}{ Número de ciclo } & \multirow{2}{*}{$\begin{array}{l}\text { Tasa de RNV } \\
\text { (IC 95\%)* }\end{array}$} & \multicolumn{4}{|c|}{ Tasa acumulada de RNV (IC95\%)* } \\
\hline & & Optima & Ajustada a edad & Ajustada a pronóstico & Conservadora \\
\hline 1 & $29,5(29,3$ a 29,7$)$ & $29,5(29,3$ a 29,7$)$ & $29,5(29,3$ а 29,7$)$ & $29,5(29,3$ a 29,7$)$ & $29,5(29,3$ a 29,7$)$ \\
\hline 6 & $17,4(15,2$ a 19,6$)$ & 78 (77,3 a 78,8) & 76,7 (76 a 77,5) & $65,3(64,8$ a 65,8$)$ & $46,8(46,5$ a 47) \\
\hline
\end{tabular}

${ }^{*}$ IC 95\%: intervalo de confianza del 95\%

\section{Conclusiones}

La tasa acumulada de RNV luego de seis ciclos de FIV fue de $65,3 \%$ para el grupo estudiado, con variaciones según edad y tipo de tratamiento. Estos hallazgos apoyan la eficacia de ampliar el número de intentos de FIV más allá de tres a cuatro ciclos.
Fuente de financiamiento: UK Medical Research Council, National Institute for Health Research y Wellcome Trust. Conflicto de interés de los autores: EI Dr Nelson participó en asesoramiento y recibió honorarios de Beckman Coulter, Besins, Ferring, Merck Serono, Merck Sharp \& Dohme y Roche.

\section{Comentario}

La probabilidad de lograr un RNV con tratamientos de FIV reiterados no es demasiado certera. Habitualmente luego de realizar tres a cuatro transferencias embrionarias sin éxito se sugiere la realización de tratamientos alternativos, con tasas de embarazo superiores, como la ovodonación. Esta práctica ha sido influenciada por estudios realizados cuando aún no se realizaba ICSI, los cuales reportaban declinación de la tasa de RNV luego del cuarto ciclo. La falla recurrente de implantación consiste en la imposibilidad de lograr embarazo luego de reiteradas transferencias en sucesivos tratamientos de alta complejidad. No existe en la bibliografía una definición universal sobre el número de embriones o transferencias necesarias para definir dicha situación ${ }^{1}$. En la última década la efectividad del congelamiento de embriones se ha incrementado de manera considerable promoviendo la transferencia de un único embrión, por lo que se sugiere que el éxito de la FIV debería calcularse como tasa de RNV por ciclo iniciado de estimulación ovárica, incluyendo todas las subsecuentes transferencias tanto del ciclo fresco como posteriores ciclos congelados$^{2}$. Dentro de los factores predictivos más importantes para lograr un RNV luego de una FIV se encuentra la edad materna, dato que se refleja en las tasas acumuladas de éxito. Mujeres menores a 40 años, e incluso aquellas entre 40 a 42 años se ven beneficiadas con sucesivos intentos, por lo que un resultado negativo o un bajo rédito ovocitario en un tratamiento previo no debería ser motivo de discontinuación. En contraste, en mujeres mayores a 42 años, la predicción de éxito en reiterados tratamientos no refleja un aumento en las tasas de RNV, por lo que en estos casos estaría justificado sugerir un tratamiento con óvulos donados ${ }^{3}$.

\section{Conclusiones del comentador}

El adecuado asesoramiento a los pacientes sobre las tasas de éxito con repetidos tratamientos de fertilidad permite por un lado evitar la discontinuación temprana de los mismos por falta de éxito y por el otro evitar el sobretratamiento con sus potenciales efectos adversos. Ajustar esos datos por edad y tratamiento ofrecido colabora con la toma conjunta de decisiones sobre costo efectividad y en especial con la difícil decisión de reemplazar gametas propias.

María Valeria Cerisola [ Servicio de Ginecología, Sección Reproducción del Hospital Italiano de Buenos Aires. maria.cerisola @ hospitalitaliano.org.ar] Cerisola MV. La tasa acumulada de recién nacido vivo aumentaría a mayor número de intentos de fertilización in vitro. Evid Act Pract Ambul. $2017 ; 20(1): 15$. Comentado de: Smith AD, y col. Live-Birth Rate Associated With Repeat In Vitro Fertilization Treatment Cycles. JAMA. $2015 ; 314(24): 2654-62$. PMID: 26717030.

\section{Referencias}

1. R. Polanzky M, y col. What exactly do we mean by 'recurrent implantation failure'? A systematic review and opinion. RBM 2014;28:409-423.

2. Luke B, y col. Cumulative birth rates with linked assisted reproductive technology cycles. N Engl J Med. 2012;366(26):2483-2491

3. Rothman KJ, y col. Volitional determinants and age-related decline in fecundability: a general population prospective cohort study in Denmark. Fertil Steril. 2013;99(7):1958-1964. 